\title{
An Effective Control Method of IP Camera Based on Fuzzy Logic and Statistical Moments
}

\author{
Muhammad Fayaz ${ }^{1}$ and Dohyeun $\mathrm{Kim}^{2 *}$ \\ 1,2 Department of Computer Engineering, \\ Jeju National University, Republic of Korea \\ ${ }^{1}$ hamaz_khan@yahoo.com, ${ }^{2}$ kimdh@jejunu.ac.kr
}

\begin{abstract}
This paper presents the IP camera control mechanism through fuzzy logic, a lot of applications of fuzzy logic in control scenarios grab our attention to control the IP camera through it. Statistical moments also play an important role for feature extraction in image processing, therefore we considered the statistical moments for extracting features from image due to its simplicity and preciseness. In the proposed work first the image capturing operation is performed, the image is captured through IP camera, after image capturing the statistical moments calculation of the capturing image is performed in order to get the feature of interest from that image. Finally, these features are fed to fuzzy logic. The output of fuzzy logic with some factor is used to control the IP camera. The results indicate that fuzzy logic is an efficient way to control IP camera and can be used in many real life applications.
\end{abstract}

Keywords: Fuzzification, IP Camera, Defuzification, Statistical Moments, Fuzzy Logic

\section{Introduction}

An internet protocol camera or IP camera is a video camera, contrasting to analog closed circuit television (CCTV) cameras has the capability of sending or receiving data through internet. The IP camera usually uses for surveillance. Axis Neteye 200 was the first centralized IP camera, it was released by Axis Communication in 1996. IP cameras may differ from one another in features and functions, video encoding (compression) schemes, available network protocols, and the API to be used by video management software. Many operations may be used in order to control the IP camera, i.e. zoom in, zoom out, rotation, etc. Here in this article we have used fuzzy logic to control IP camera [1-2]. In recent age the IP cameras usage in various places have been significantly increased, such as in movie theatres, Universities, markets, malls, etc. These applications of IP camera grasp the attention of the user

First, the term fuzzy logic introduced in 1965 by Lotifi Zadeh, is a procedure of several valued logic, in fuzzy logic the values of variables can be any real value between 0 and 1 . In Boolean logic, the truth values of variables may be either 0 or 1, these are called crisp values. It is normally used in order to handle the concept of partial truth, where the truth value may be in range of completely true and completely false. The fuzzy logic has four main components, namely fuzzification, inference engine, knowledge base and defuzzification [3]. In order to construct a fuzzy system, the first work that is carried is to define linguistic variables and terms then the membership function are constructed and ranges are assigned to membership functions. Rules construction is an important phase of fuzzy logic design, rules are carried out from membership functions of input/ outputs variables, the selection membership function for output variable in rules is subjective and depends on the observer or designer. The next step is fuzzification in order to convert crisp data into fuzzy data by using membership functions. Normally the rules in the fuzzy logic are in the rule base, hence the rules are evaluated in the rule base, the result is 
combined from the rule base and at the last the defuzzification is applied to these rules in order to get the crisp output from fuzzy values [4-5]. The architecture of the fuzzy logic is illustrated in Figure 1. The fuzzy is normally used for controlling purposes, fuzzy logic has the capability to control the machines and consumer products, i.e. air conditioner, dryers, heaters. It doesn't always give the exact value, but always gives us the approximate result. The fuzzy logic is normally used to deal with uncertainty [6]. There are four main components of fuzzy logic namely fuzzification, inference, composition and defuzzification. The fuzzification process uses the membership function to convert the crisp inputs to the fuzzy input. There could be one or more input variable in fuzzy logic and each variable may have many membership functions, each membership function may have different ranges. The defuzzification also uses the membership functions to convert the fuzzy values to the crisp output. There are different types of membership functions, such as bell shaped membership functions, Gaussian membership function, triangular membership function and sigmoid membership functions [7].

In this paper, we propose the IP camera control mechanism through fuzzy logic in control scenarios grab our attention to control the IP camera through it. The rest of the paper is organized as follows: Section 2 extant the literature review, Section 3 shows the proposed work, experimental results and discussion is given in detail in Section 4 and the conclusion is given in Section 5.

\section{Related Work}

In this work fang et al. proposed a system [10], the proposed system comprises of main three steps namely object tracking (infant), infant tracking, initialization, and PT IP camera control. For background subtraction the codebook algorithm is used in order to extract the input frames and to first construct a tracking feature. After infant extraction, then the tracking feature model is used for tracking infant. As the infant changes the position or posture the feature tracking model is updating accordingly. This system also has the prediction capability and it also predicts the infant's actions and controls the PT IP camera movement in order to escape from crawling infant or to cross the boundary of the monitoring scope, the retracing mechanism is used.

Nowadays the IP camera is extensively used for security purposes however there is a lot of space to work on these security devices. In this paper Ali et al. [11] investigated the cloud-based IP cameras security. There is numerous applications of these devices in many research spaces, such as secure multimedia, network security and cloud security. In this work the investigation has been done for traffic generation wireless camera for average home users. The security provision taken by IP cameras manufactures have been explored in this study and many security and privacy issues have also been investigated.

In this work Junying et al. [12] designed a real time face recognition system, the proposed system is based on IP camera and image processing algorithms. The OpenCV and $\mathrm{C}++$ programming have been used to way out it. This work has been divided into three parts, namely preview module, register module and recognition module. For real time face recognition the AdaBoost algorithm has been used which carry out the real realtime face detection and join the nose detection for secondary screening. Next for texture feature extraction from detected face image, texture feature extraction algorithm is used. At the end the face recognition is done that is based on image sets. The testing results show that the suggested method is effective for processing video in real-time and provide good results on face recognition and detection.

\section{Proposed Control Method of IP Camera Based on Fuzzy Logic}

The proposed configuration for controlling the IP camera by using fuzzy logic is illustrated in Figure 1. In the proposed methodology, first the feature extraction module request to image module for getting an image. The image module gets an image from the 
IP Camera, after getting the image from image module, the feature extraction stage calculates the first two statistical moments (mean, variance). The values of these statistical moments are then input to fuzzy logic. The fuzzy logic gets the crisp input and provides crisp output and some action are performed according to the fuzzy logic on that IP camera.

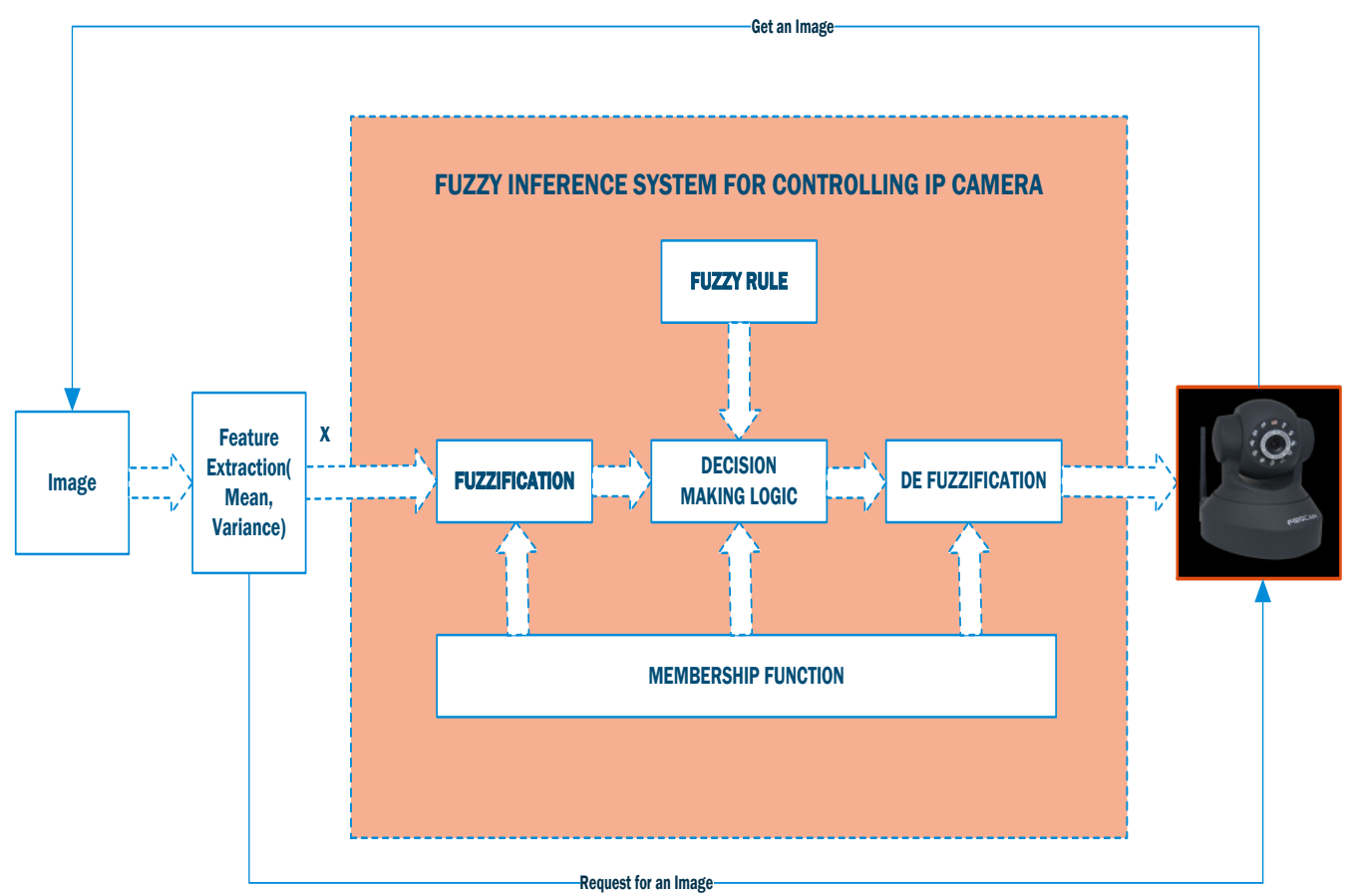

Figure 1. Proposed Configuration of Control Method of IP Camera Based on Fuzzy Logic

We use the triangle membership function included a great significant and the most commonly used membership function. Therefore, we have used triangular membership functions in the proposed fuzzy logic. The triangular function defined by a lower limit a, an upper limit $\mathrm{b}$, and a value $\mathrm{m}$, where $\mathrm{a}<\mathrm{m}<\mathrm{b}$. Equation 1 is triangular membership function specification.

$$
\mu_{\mathrm{A}(\mathrm{x})}=\left\{\begin{array}{cr}
0 & \mathrm{x} \leq \mathrm{a} \\
(\mathrm{x}-\mathrm{a}) /(\mathrm{m}-\mathrm{a}) & \mathrm{a}<\mathrm{x} \leq \mathrm{m} \\
(\mathrm{b}-\mathrm{x}) /(\mathrm{b}-\mathrm{m}) & \mathrm{m}<\mathrm{x}<\mathrm{b} \\
0 & \mathrm{x} \geq \mathrm{b}
\end{array}\right.
$$

The rule matrix is used for description of fuzzy sets and fuzzy operations; it defines it in the form of conditional statements. An if-then rule can be defined as: 


\section{If $x$ is $\mathrm{A}$ then $y$ is $\mathrm{Z}$}

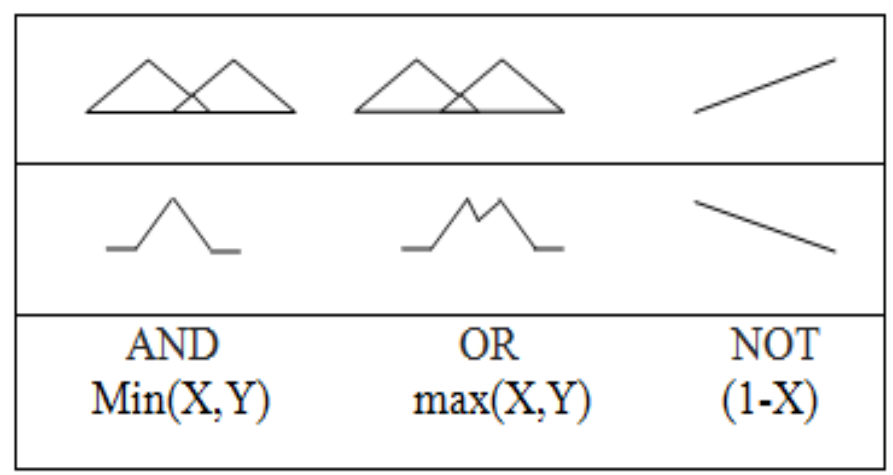

Figure 2. Graphical Interpretation of Fuzzy Operators

For combining more than one input the fuzzy operations used are: $A N D=\min , \mathrm{OR}=\max$ and NOT= additive complement. The Figure 2 shows the geometrical demonstration of fuzzy operations [8].

Where A represents the set of conditions that have to be satisfied and $\mathrm{Z}$ is a set of consequences that can be inferred. This is a simple graphical tool that is used to map the fuzzy logic rules. The working mechanism of the fuzzy logic is given in Figure 3. Figure 4 shows the input, output and different components of fuzzy logic.

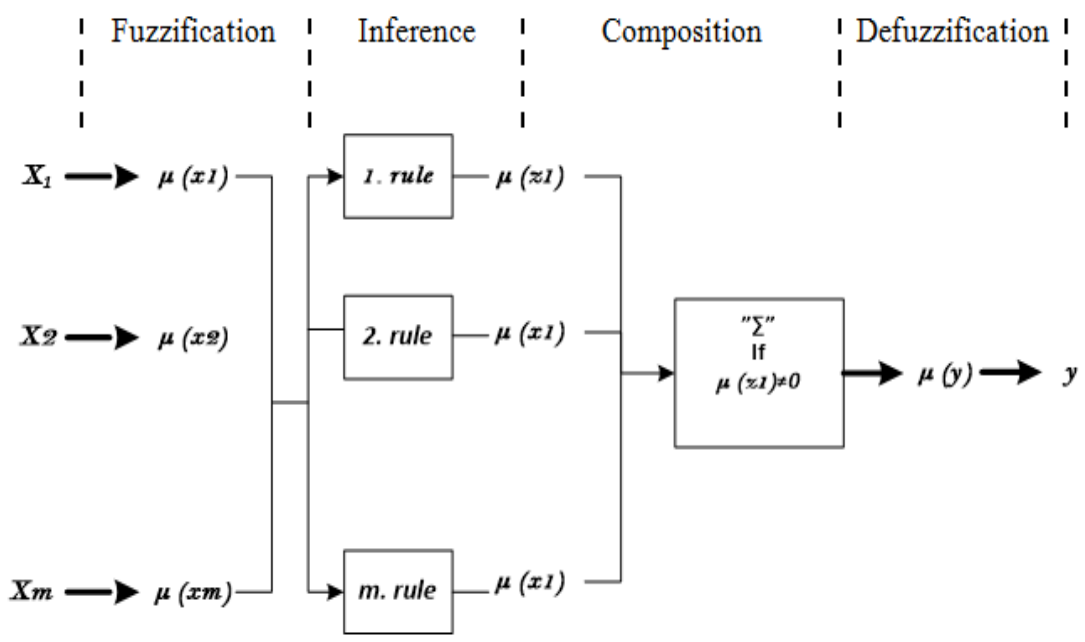

Figure 3. Fuzzy Logic Working Mechanism

The statistical moments also play an important rule for finding nature of the data. In fuzzy logic the input may be single or few values, as we can't exceed the input variables, because by increasing the input variables the rules will be also increased exponentially, hence the computation complexity will also be increased exponentially causing problem. On another side thorough IP camera we can capture images or record videos. Each image has thousands of pixel values; hence in the proposed work we have used statistical moments to find the nature of an image. There are four statistical moments that are mean, variance, skewness and kurtosis [10], here in this work we have used the first two statistical moments, namely mean and variance(standard deviation) which are represented in equation 2 and 3 respectively. 


$$
\begin{gathered}
\mu_{X}=\bar{X}=\frac{1}{n} \sum_{i=1}^{n} X_{i} \\
\sigma_{X}=\sqrt{\frac{1}{n}\left\{\sum_{i=1}^{n} X_{i}^{2}-\frac{1}{n}\left(\sum_{i=1}^{n} X_{i}\right)^{2}\right\}}
\end{gathered}
$$

Where $i=1$ to $n$, represents the pixels value of an image.

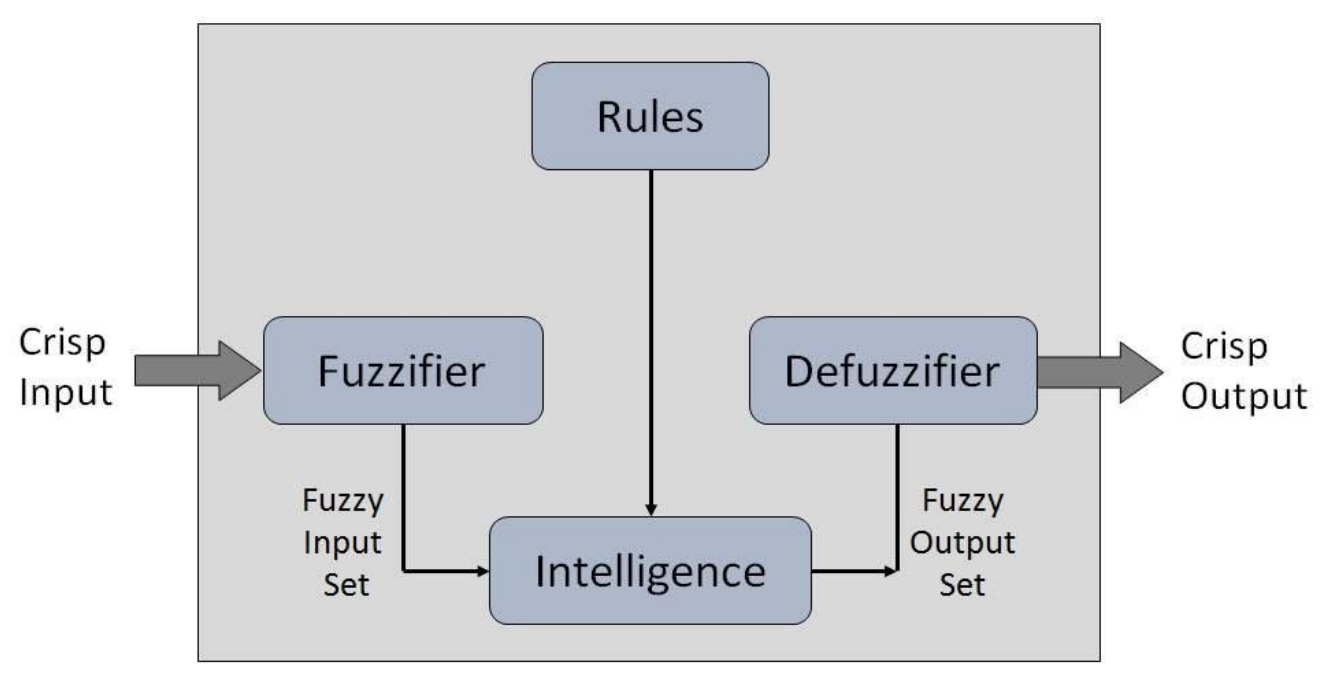

Figure 4. Fuzzy Logic Systems Architecture

The sequence diagram of the proposed configuration for controlling IP camera through fuzzy logic is illustrated in Figure 5. First the input module gets the input from the feature extraction stage, the inputs are then pass to fuzzification module, the rule employment, implication and aggregation is carried out in fuzzy inference module. At last we need crisp output, then on these fuzzy out the centroid method is applied on defuzzification module for crisp output and base on these outputs the image is zooming in or out.

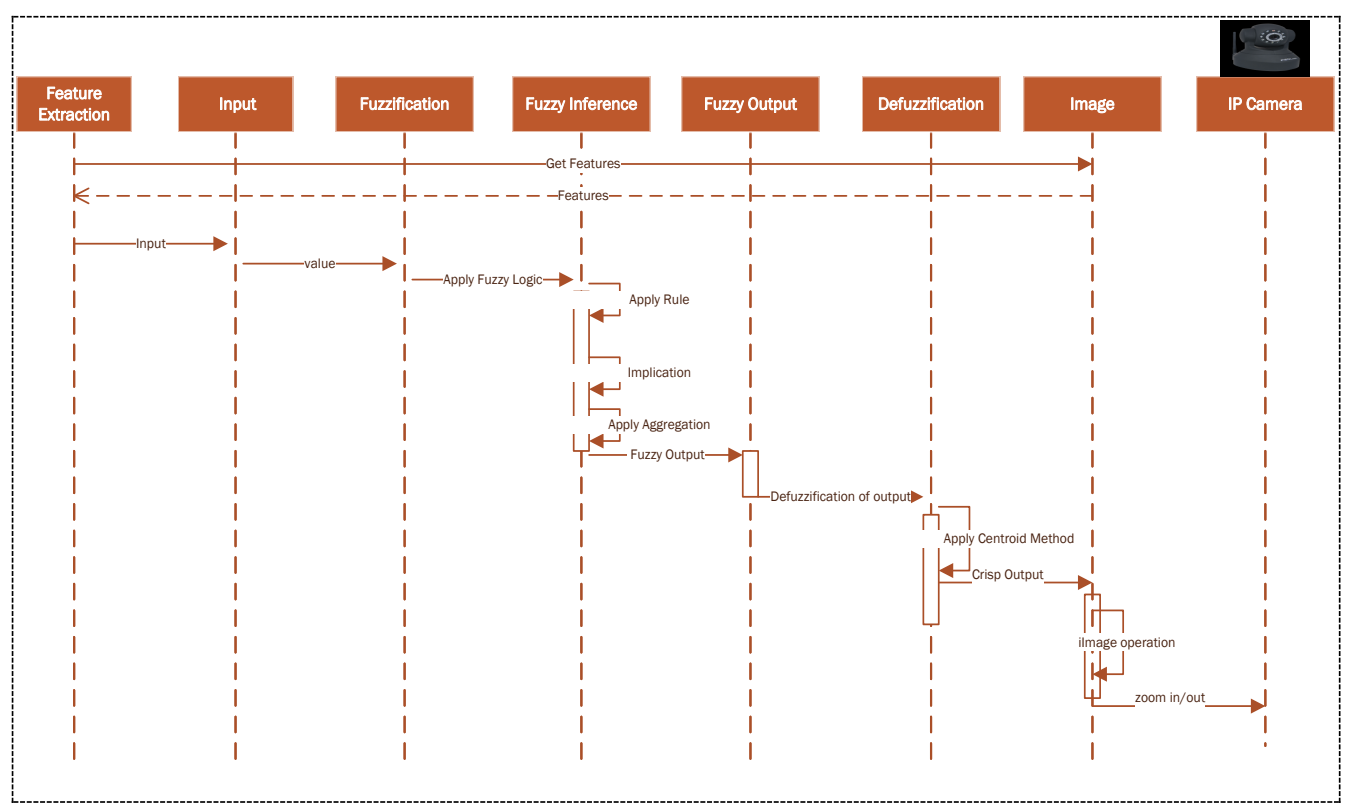

Figure 5. Sequence Diagram for Proposed Methodology 


\section{Implementation and Experimental Results}

All the code is done in MATLAB R2015a version using an Intel core i5 system on running windows 7, for fuzzy logic we have used fuzzy logic toolbox, created membership functions, defined its ranges, defined input/output variables, etc. Figure 6 shows the fuzzy inference system and membership functions. First we captured an image by using IP camera, then the statistical mean and variance are calculated of that image's pixel values. As the images normally taken through IP camera are RGB images and an RGB image comprises of three channels that are red, green and blue. In this work we have considered the red channel for mean and variance calculation of image pixels.

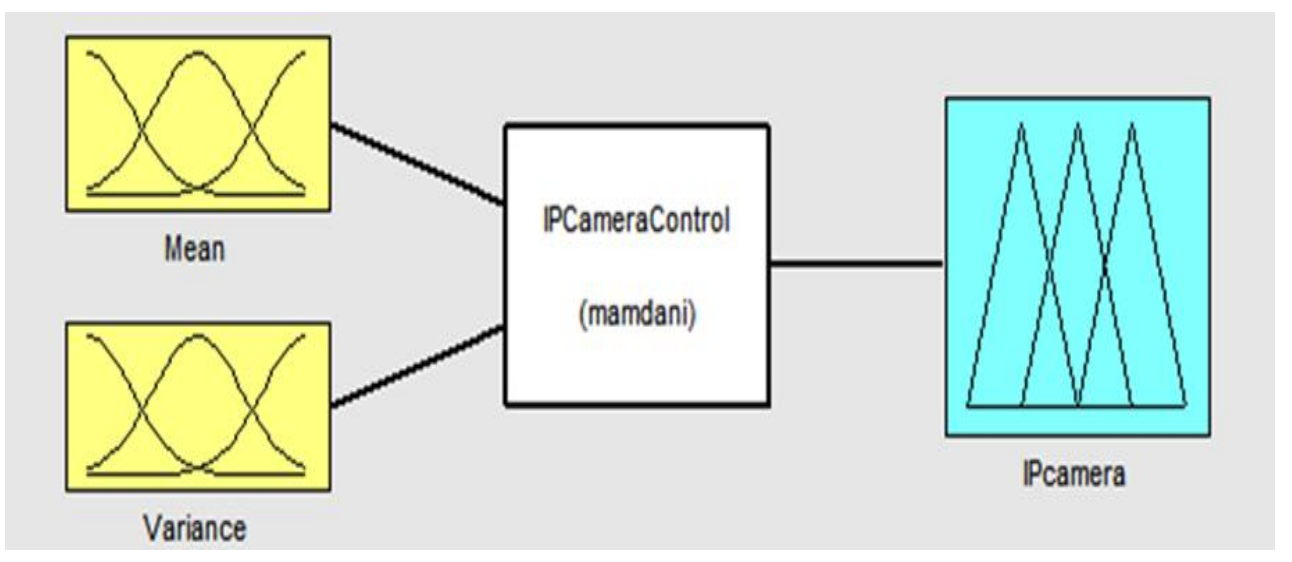

Figure 6. Proposed FIS for IP Camera Control

The proposed fuzzy Inference system for IP camera control has two Input variables, namely mean and variance and one output variable that is IP camera. There are five membership functions in each variable. The ranges of the input variables are fixed on mean of the pixel values of an image. The range of the output value is desired size of an image. The input/output membership functions are given in Figure 7.

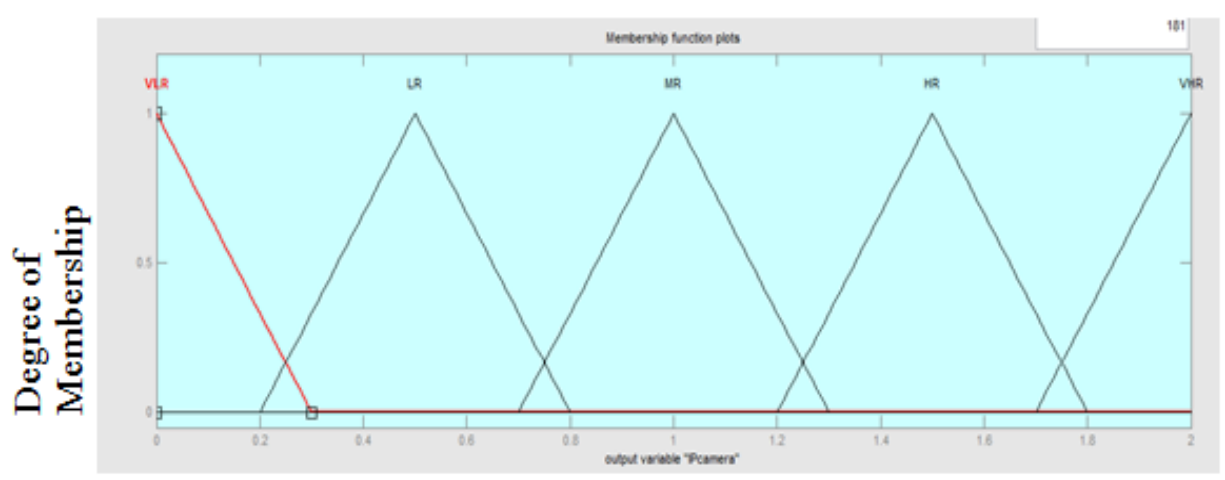

\section{Figure 7. Input/Output Membership Functions of Proposed Fuzzy Logic for IP Camera Control}

Rule design is the most important part of fuzzy logic, the rule design method is subjective and in fuzzy logic one can define rules according to his/ her requirement. Rules are mainly defined by the experts for applications. In the prosed system the general rule mechanism has been carried out. The user can set it according to their needs. Table shows the rules used in the proposed fuzzy logic for IP camera control and Figure 8 shows the rule viewer. 
Table 1. Rules for IP Camera Control Fuzzy Logic

\begin{tabular}{|c|c|c|c|c|c|}
\hline $\begin{array}{c}\text { Mean } \\
\text { Variance }\end{array}$ & VL & L & M & H & VH \\
\hline VL & VL & VL & L & M & M \\
\hline L & VL & L & M & M & H \\
\hline M & L & M & M & H & VH \\
\hline H & M & M & H & VH & VH \\
\hline VH & M & H & VH & VH & VH \\
\hline
\end{tabular}

The rule viewer shows the implication process entirely. It is possible in rule viewer to do movement around the line, and according to that line movements the new output are carried out.

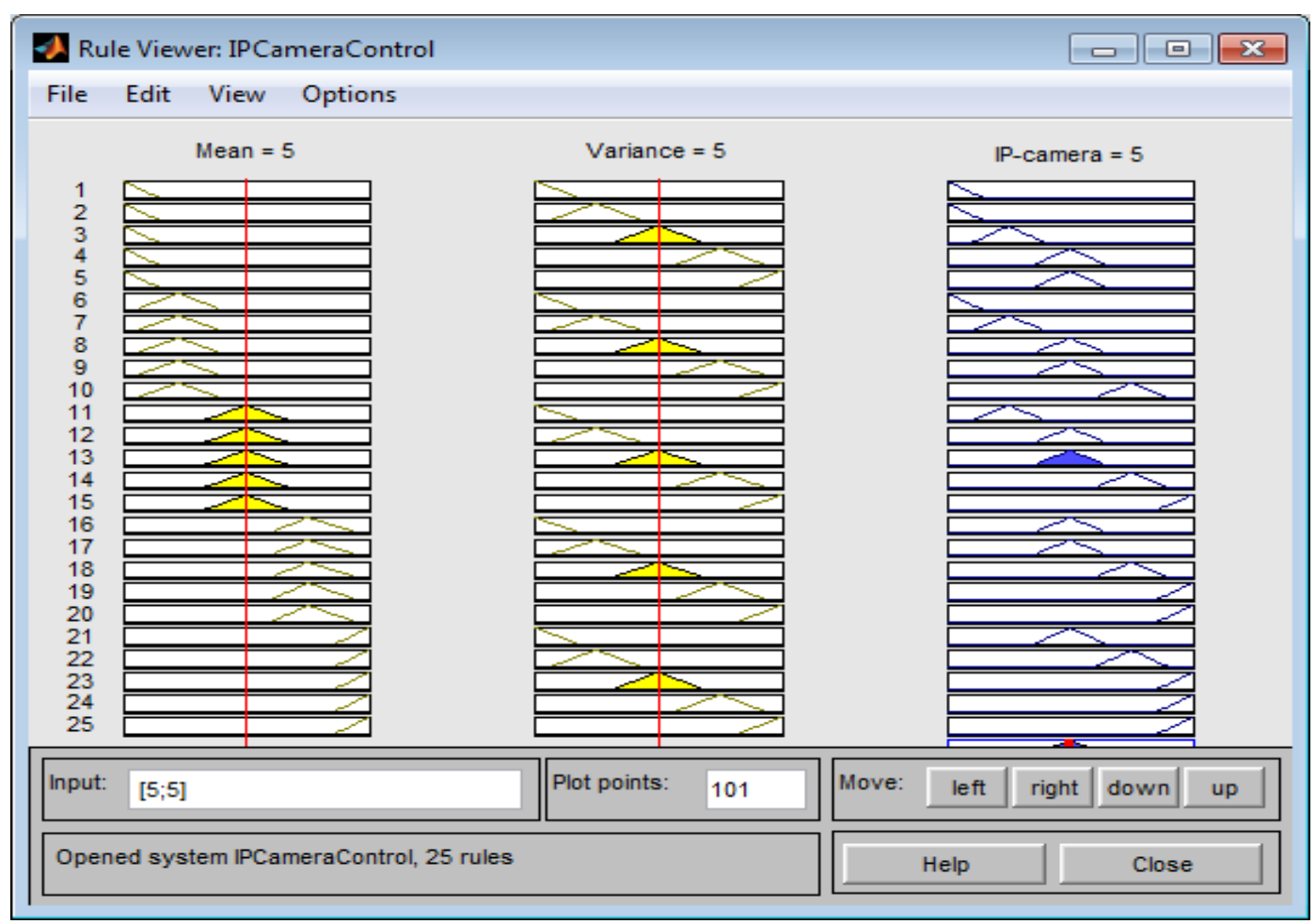

Figure 8. Rule Viewer of the IP Camera Control Fuzzy Logic

The rule editor is used to edit the set of rules, these rules defines the attitude of the system. The editor has made it easy to define the rule for the system. Figure 9 shows the rule editor, input membership functions' terms, output membership functions and different type of connector used in rule editors.

The zoom out and zoom in operations on the IP camera is illustrated in Figure 10. According to the output of the fuzzy logic, the IP camera has been zoom in or zoom out. In Figure 10.a, we can see the original view of IP camera, Figure 10.b is the zoom in view of the IP camera by using the fuzzy logic and Figure 10.c is the zoom out view. 
Rule Editor: IPCameraControl

1. If (Mean is VL) and (Variance is NI) then (IP-camera is VLR) (1)

2. If (Mean is VL) and (Variance is $L I)$ then (IP-camera is VLR) (1)

3. If (Mean is VL) and (Variance is MI) then (IP-camera is LR) (1)

4. If (Mean is VL) and (Variance is $\mathrm{HI}$ ) then (IP-camera is MR) (1)

5. If (Mean is VL) and (Variance is VHI) then (IP-camera is MR) (1)

6. If (Mean is $L$ ) and (Variance is NI) then (IP-camera is VLR) (1)

7. If (Mean is $L$ ) and (Variance is $L I)$ then (IP-camera is $L R$ ) (1)

8. If (Mean is $L$ ) and (Variance is MI) then (IP-camera is MR) (1)

9. If (Mean is $L$ ) and (Variance is $\mathrm{HI}$ ) then (IP-camera is MR) (1)

10. If (Mean is $L$ ) and (Variance is VHI) then (IP-camera is HR) (1)

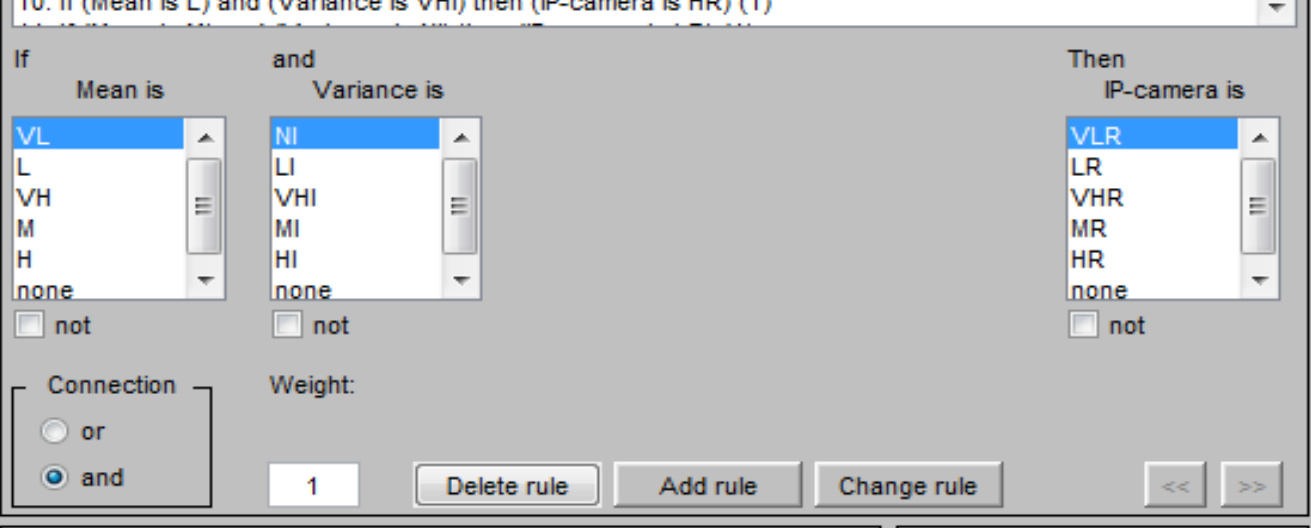

FIS Name: IPCameraControl

Figure 9. Rule Viewer of the IP Camera Control Fuzzy Logic

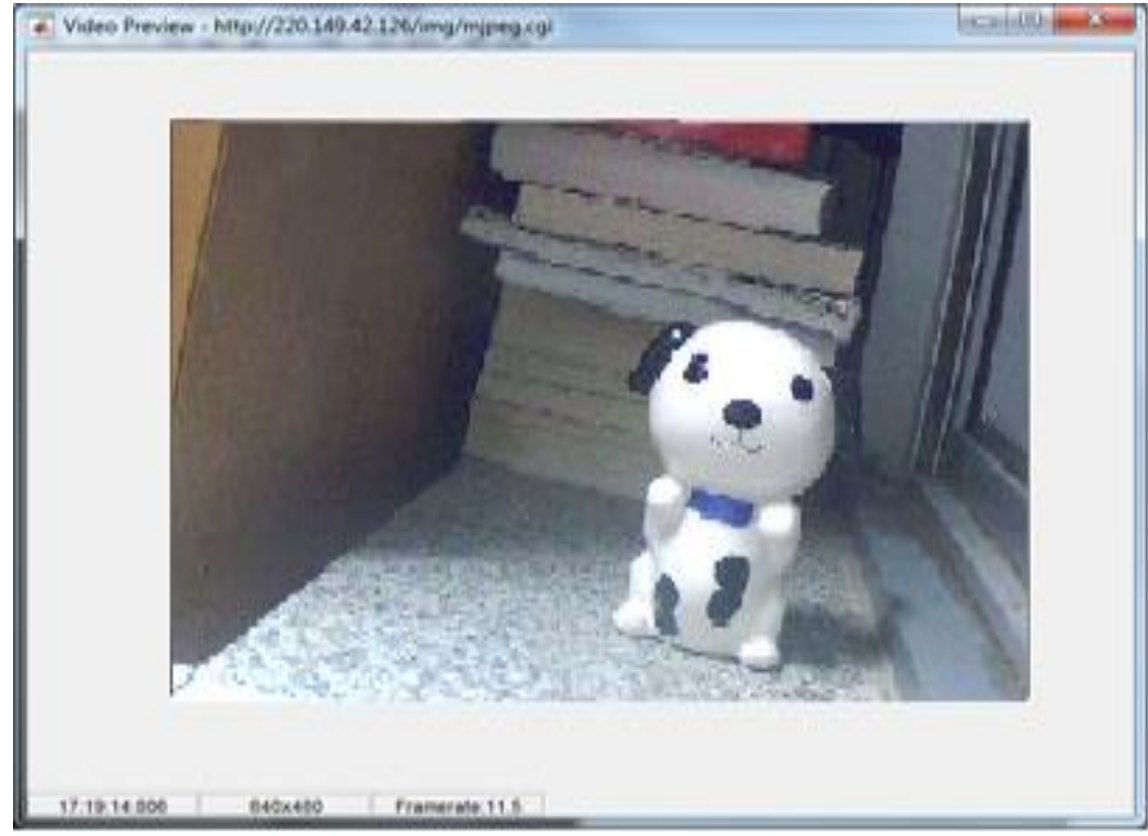

(a) Normal View 


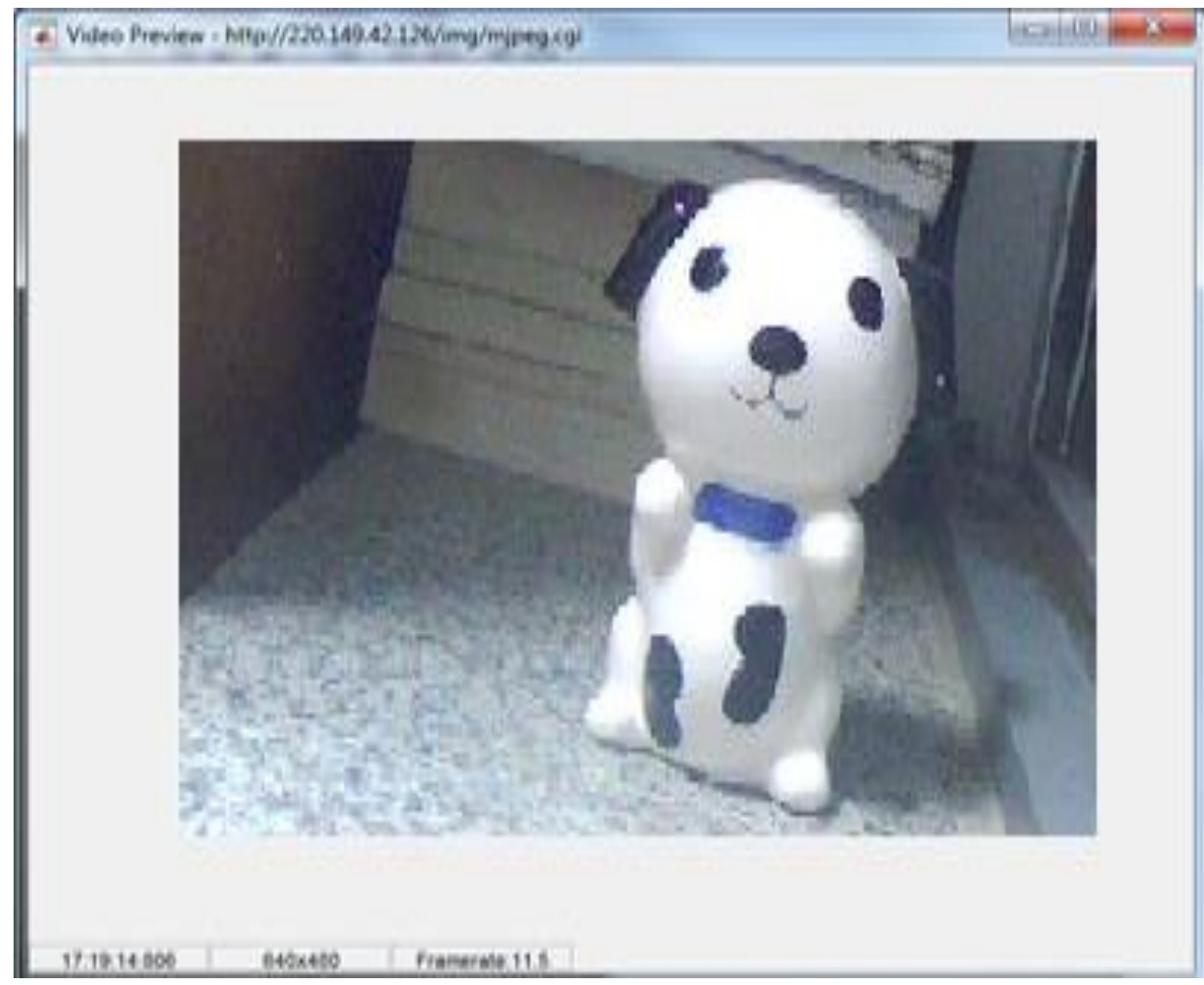

(b) Zoom In

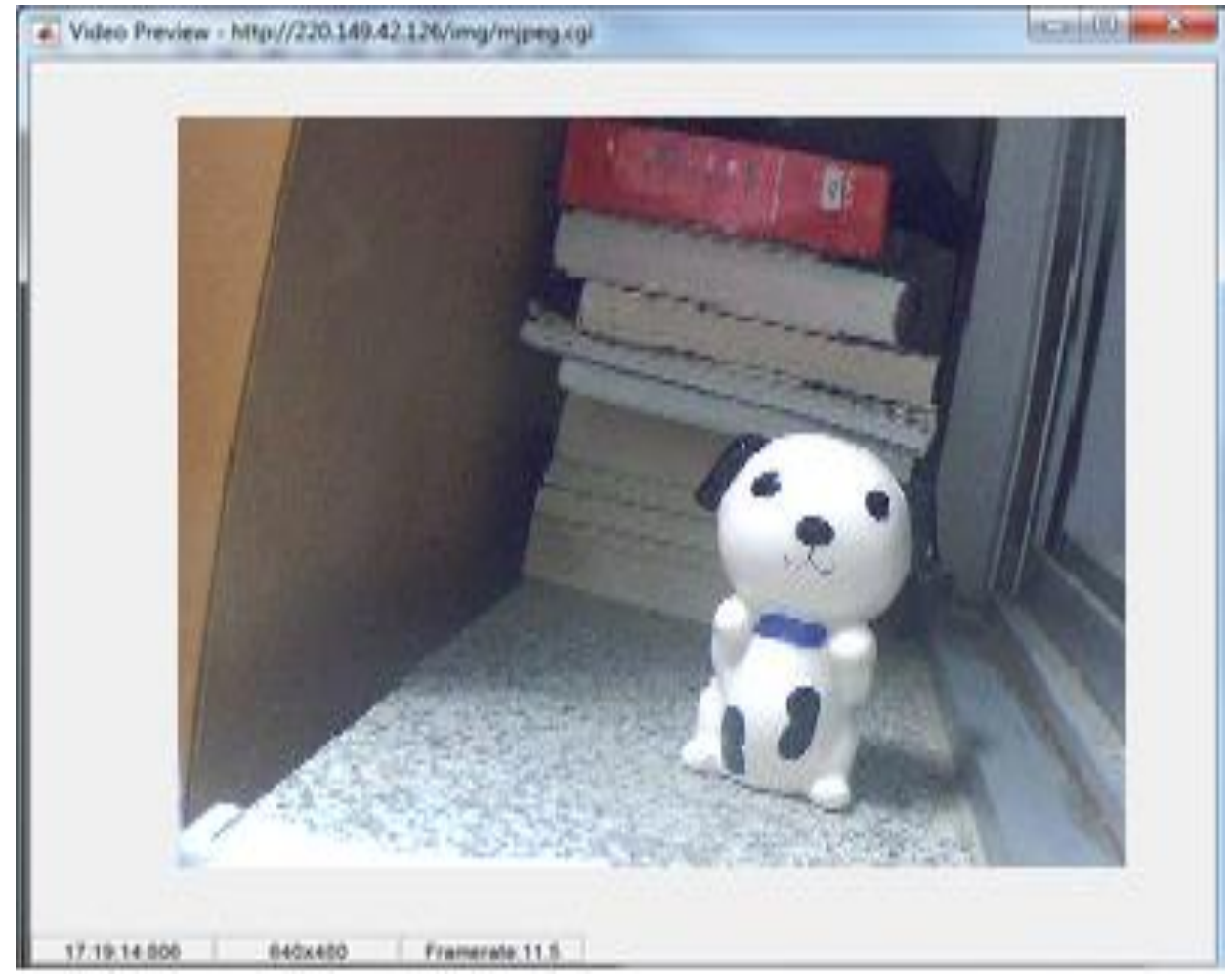

(c) Zoom Out

Figure 10. Control Results of IP Camera 


\section{Conclusion and Future Work}

In this study the fuzzy logic is discussed in detail, each component's working and mechanism of fuzzy is explained in detail, some applications of fuzzy logic are also discussed. The detail literature review on IP camera is also carried out. In this paper, we have made an attempt to control the IP camera through fuzzy logic, numerous operations can be carried out on IP camera, but in this work we have done the zoom in and zoom out operations. The results are promising for tackling the IP camera control through fuzzy logic. The fuzzy logic performs these operations in efficient way. The basic purpose of this research was to control IP camera efficiently. In future we would like to control more operations of IP camera like rotation, movements, etc. through fuzzy logic.

\section{Acknowledgment}

This research was supported by Basic Science Research Program through the National Research Foundation of Korea(NRF) funded by the Ministry of Education, Science and Technology (2015R1D1A1A01060493) and this research was supported by the MSIP(Ministry of Science, ICT and Future Planning), Korea, under the ITRC(Information Technology Research Center) support program (IITP-2016-H8501-16-1017) supervised by the IITP(Institute for Information \& communications Technology Promotion), Correspond author : DoHyeun Kim

\section{References}

[1] Y.-H. Lin, "The complete guide to physical security", CRC Press

[2] K. Otsuki, K. Nishiwaki and H. Sasaki, "Wireless camera network system and wireless image capturing device", Google Patents, (2004).

[3] R. R. Yager and L. A. Zadeh, "An introduction to fuzzy logic applications in intelligent systems", Springer Science \& Business Media, vol. 165, (2012).

[4] Y. John and R. Langari, "Fuzzy logic: intelligence, control, and information", Publish by Dorling Kindersley, India, (1999), pp. 379-383.

[5] C.-C. Lee, "Fuzzy logic in control systems: fuzzy logic controller. II", IEEE Transactions on systems, man, and cybernetics, vol. 20, (1990), pp. 419-435.

[6] G. Klir and B. Yuan, "Fuzzy sets and fuzzy logic", Prentice hall New Jersey, vol. 4, (1995).

[7] J. L. Castro, "Fuzzy logic controllers are universal approximates", IEEE Transactions on systems, man, and cybernetics, vol. 25, (1995), pp. 629-635.

[8] M. D. Symans and S. W. Kelly, "Fuzzy logic control of bridge structures using intelligent semi-active seismic isolation systems", Earthquake engineering \& structural dynamics, vol. 28, (1999), pp. 37-60.

[9] M. Nixon, "Feature extraction \& image processing", Academic Press, (2008).

[10] C.-Y. Fang, C.-S. Lo, S.-H. Ho, S.-H. Chuang and S.-W. Chen, "A Vision-Based Infant Monitoring System Using PT IP Camera”, Computer, Consumer and Control (IS3C), 2016 International Symposium, pp. 279-282.

[11] A. Tekeoglu and A. S. Tosun, "Investigating Security and Privacy of a Cloud-Based Wireless IP Camera: NetCam", 24th International Conference on Computer Communication and Networks (ICCCN), (2015), pp. 1-6.

[12] J. Gan, X. Wang and Y. Zhai, "A Real-Time Face Recognition System Based on IP Camera and Image Sets Algorithm", Ubiquitous Intelligence and Computing and 2015 IEEE 12th Intl Conference on Autonomic and Trusted Computing and 2015 IEEE 15th Intl Conference on Scalable Computing and Communications and Its Associated Workshops (UIC-ATC-ScalCom), 2015 IEEE 12th Intl Conference, pp. $1524-1528$. 


\section{Authors}

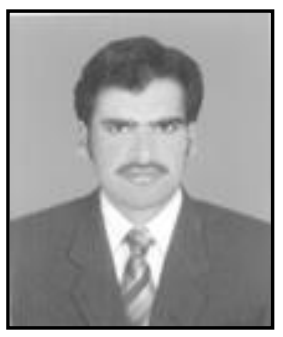

Muhammad Fayaz Abdul Ahad, is currently perusing Ph.D. in Department of Computer Engineering, Jeju National University, Republic of Korea. He received his MS in Computer Science from SZABIST, Islamabad, Pakistan in 2014. He did MSc in Computer Science from University of Malakand, Chakdara KPK, Pakistan.

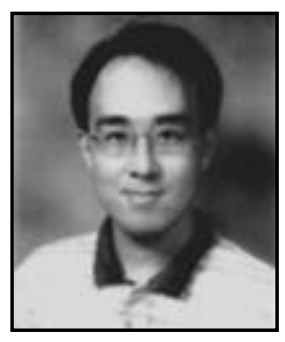

Do-Hyeun Kim, He received the B.S. degree in electronics engineering from the Kyungpook National University, Korea, in 1988, and the M.S. and Ph.D. degrees in information telecommunication the Kyungpook National University, Korea, in 1990 and 2000, respectively. He joined the Agency of Defense Development (ADD), from Match 1990 to April 1995. Since 2004, he has been with the Jeju National University, Korea, where he is currently a Professor of Department of Computer Engineering. From 2008 to 2009, he has been at the Queensland University of Technology, Australia, as a visiting researcher. His research interests include sensor networks, M2M/IOT, energy optimization and prediction, intelligent service, and mobile computing. 
International Journal of Control and Automation Vol.10, No.4 (2017) 